\author{
Jacques Henrard \\ Facultés Universitaires, \\ B-5000 Namur, Belgium
}

\begin{abstract}
Almost every aspect of the analytical theory of the motion of the Moon has been reinvestigated lately. This paper is a review of these investigations.

The improvement upon the I.L.E. (the best known earlier theory based upon the work of Brown) is spectacular, but it is still too early to assess the exact value of these theories with respect to numerical integration.
\end{abstract}

\title{
INTRODUCTION
}

For the last ten years, there has been a new interest and new results about analytical theories of the Moon.

The new interest evidently comes from the challenge presented by the new types of observations, especially the laser ranging.

The new results were made possible by the techniques of algebraic manipulations on computers. These techniques are still in their infancy and we may predict that in the long run they will have a very deep impact on Celestial Mechanics.

After the monumental work of Delaunay (1867) and that of Brown (1908), the theory of the motion of the Moon was dead as a research field for two reasons : the accuracy of Brown's solution was for some time superior to the accuracy of the observations ; the work necessary to improve upon this solution (without the help of a computer) was of the order of a scientist's lifetime.

With the coming of the space age, it became obvious that the first reason was no longer valid (see, for instance, Garthwaite et a1. 1970). Even the modern adjustment of Brown(s theory, known as I.L.E. (Eckert et a1. 1954) was not accurate enough to be of any help in the reduction of the data of spacecraft landed on the Moon. 
It was later shown that computers could be used to perform the tremendous algebraic computations involved in analytical theories (Barton, 1967, Eckert et a1.1966, Deprit et a1. 1970 a and b, 1971).

The construction of a theory of the motion of the Moon at the level of accuracy of the new observations thus became a challenge for Celestia1 Mechanicians.

For several years, the work in this field was almost exclusively directed towards the solution of the Main Problem (three body problem Earth, Moon and Sun) although it was suspected that the largest inaccuracies were coming from the treatment of the planetary perturbations.

The reasons for this are twofold. First, tools, such as algebraic manipulators and perturbation schemes, had to be developed and experimented on a simplified problem. Secondly, the treatment of the planetary perturbations require a very accurate tube of solutions of the Main Problem (hence a nominal solution and its partial derivatives with respect to the internal parameters.

We will review in Section 2 the present status of the solution of the Main Problem.

In the last few years, new results have been published about perturbations by the oblateness of the Earth (and Moon) and by the Planets. They will be described in Secion 3 .

These new results make it possible now to realistically test the accuracy of the new analytical theories (Chapront and Chapront-Touzé : these proceedings). These preliminary tests are very encouraging.

\section{THE MAIN PROBLEM}

All the solutions are obtained as Fourier series in the Delaunay angles : $D, 1^{\prime}, 1, F$. They differ according to their treatment of the coefficients of these series.

Purely analytical solutions have their coefficients expanded as Taylor series in the parameters $m$ (ratio of the mean motion of the Sun and Moon), e (eccentricity of the Moon), $Y$ (sine of half the inclination) and $e^{\prime}$ (eccentricity of the Sun). The convergence of these series with respect to the parameter $m$ is very poor. A significant example of it is the A.L.E. (Analytical Lunar Ephemeris) (Deprit 1971 and Henrard 1973). The expansions were carried to order 10 in e and $\gamma$ and order 24 in $\mathrm{m}$.

Partly analytical solutions give a numerical value to the parameter m and expand the coefficients in power series of $e, e^{\prime}$, and $\gamma$. The E.L.E. solution (Gutzwiller, 1979) has been started by M.J. Eckert and 
S. Bellesheim and completed after the death of W.J. Eckert by

M.C. Gutzwiller and S. Bellesheim. The expansions have been carried to the order 6 in $e, e^{\prime}$, and $\gamma$. A more recent solution by Schmidt (1980) pushes the expansions to order 10.

Semi-analytical solutions bypass the poor convergence with respect to $m$ and to a lesser extent with respect to the other parameters e, $e^{\prime}$, and $\gamma$ not by giving a numerical value to one or more of these parameters, but by expanding the coefficients around nominal values which are closer to the real values. The expansions are in power of increments $\delta \mathrm{m}, \delta \mathrm{e}, \delta \gamma, \delta \mathrm{e}^{\prime}$. Such a solution (known as SALE Semi-Analytical Lunar Ephemeris) has been developed by the author (Henrard, 1979).

Semi-numerical solutions give numerical values to the parameter. To be useful, the solutions (as well as the partly analytical solutions) should be completed by at least the first partial derivatives with respect to the parameters which have been given a numerical value.

This is not the case for the Eckert-Smith solution (1966), but this is the case for the E.L.P. (Ephéméride Lunaire Parisienne) of ChaprontTouzé (1980).

Comparisons have been made between those theories and for two of them (SALE and E.L.P.) comparisons have been made with numerical integration (Kinoshita 1982 a and b).

From these comparisons, it results that E.L.P. and Schmidt's solution are accurate, at least at leve 1 of $10 \mathrm{~cm}$. To assess if one of them is more accurate than that, the truncature level at which they are published should be lowered.

By comparison, SALE has discrepancies of the order of 1.5 meters, A.L.E. and E.L.E. of the order of 10 meters and I.L.E. of the order of 30 meters.

\section{CORRECTIONS TO THE MAIN PROBLEM}

The corrections are mainly due to the shape of the Earth and to the planets. The effects of the planets is usually divided into direct, indirect and secular effects. Relativity corrections, the effects of the shape of the Moon and of tides and cross products between all those corrections should also be considered.

The effect of the shape of the Earth has been investigated up to the second-order in $J_{2}$ by the author (Henrard 1981) and up to the firstorder by Chapront-Touzé (1982). For the first-order, the two solutions agree to a few centimeters. Second-order terms are of the order of 6 meters, while nutation effects (neglected by Henrard) are of the order of 4 meters in Chapront-Touzé. 
By comparison, discrepancies between these solutions and Brown's solution used in I.L.E. are of the order of 42 meters.

The effect of the planets is certainly the most difficult part in the theory of the motion of the Moon. This is due to the fact that with each planet we introduce a new frequency to the basic four frequencies of the Main Problem and these frequencies can combine to generate very long-period terms, which are very difficult to compute.

On top of that, the Fourier series expansions of the distance Earthplanet are poorly convergent at least for Venus and Mars and produce many terms in the solution.

Direct perturbations take into account the effects of the planets directly upon the Moon. A first-order model (assuming the planets on elliptic orbits around the Sun) has been investigated by Standaert (1982 a, b and c) and by Chapront and Chapront-Touzé (1982). Standaert gives a second-order solution of this mode1 while Chapront and Chapront-Touzé propose a first-order solution.

At the first-order, the largest periodic terms in the difference between the two solutions have an amplitude of 5 meters (for a period of 5000 years) and 1.5 meters (for a period of 500 years). The next one is down to an amplitude of 0.6 meters. This is quite an improvement when compared to Brown's solution, which shows several discrepancies of the order of 100 meters (the largest one having an amplitude of 1300 meters).

The second-order corrections of Standaert contains several terms with an amplitude of 7 meters. Together they can amount to a correction of 60 meters.

For the indirect and the secular perturbations which take into account the effects of the planets upon the center of mass of the system Earth-Moon, we have only the solution by Chapront and Chapront-Touzé (1982) which proposes corrections on Brown's solution of the same order as for the direct perturbation.

Although we do not have a specific check on the accuracy of this part of the solution, we do know that it is valid at least at the order of 10 meters because of the comparison of the full solution with numerical integration (see Chapront's paper in these proceedings).

Some of the other perturbations have also been investigated. Brumberg (1972) and Lestrade (1982) have proposed corrections due to relativity. Henrard (1981) has proposed corrections due to the shape of the Moon. Chapront and Chapront-Touzé have introduced tidal effects and firstorder corrections to the model of direct planetary perturbations (which induce a kind of external second-order correction on the motion of the Moon, by contrast with Standaert's internal second-order corrections mentioned above. 


\section{CONCLUSIONS}

As exemplified by the paper of Chapront and Chapront-Touze in these proceedings, analytical theories are now able to reproduce realistically the motion of the Moon with an accuracy of several meters, an improvement by a factor of 100 or 1000 upon the I.L.E.

This accuracy can still be improved somewhat by the inclusion in the theory of several second-order effects which have not yet been computed.

An improvement by a factor of 10 to 100 which would make analytical theories more than competitive with numerical integration would still require a major effort.

Indeed, the number of terms in the theory (mainly from planetary perturbations) increase drastically when the level of truncation is lowered (see Standaert 1982).

\section{REFERENCES}

Barton, D.:1967, Astron.J., 72, 1281 .

Brown, E.W.:1908, Mem.Roy.Astro.Soc. 59, 1 .

Brumberg, V.A.:1972, Relativistic Celestial Mechanies, Manka, Moscow (in Russian).

Chapront, J., Chapront-Touzé, M.: 1982a, Proceedings of the Conference on Analytical Methods and Ephemerides, Celestial Mechanics, $26,83$.

Chapront, J., Chapront-Touzé, M.: 1982b, these proceedings, p. 257.

Chapront-Touzé, M.: 1980, Astron. Astrophys. 83, 86.

Chapront-Touzé, M.: 1982, Proceedings of the Conference on Analytical Methods and Ephemerides, Celest. Mech. 26, 53, 63.

Chapront-Touzé, M., Chapront, J.: 1980, Astron.Astrophys. 91, 233.

Chapront-Touzé, M., Henrard, J.: 1980, Astron.Astrophys . 86, 221.

Delaunay, Ch.: 1867, Mem.Acad.Sci. Paris 29.

Deprit, A.:1971, Esro-Cers Scient. and Tech.Rev. 3, 77 .

Deprit, A., Henrard, J., and Rom, A. : 1970a, Science 168, 1569.

Deprit, A., Henrard, J., and Rom, A. : 1970b, Astron. J. 75,747.

Deprit, A., Henrard, J., and Rom, A. : 1971, Astron. J. 76, 269.

Eckert, W.J., Jones, R., and Clark, H.K.: 1954, Improved Lunar Ephemeris, U.S. Gov. Printing Office.

Eckert, W.J., Smith, H.F. : 1966, Astron.Papers of the American Ephemeris 19, Part II.

Eckert, W.J., Walker, M.J., and Eckert, D.: 1966, Astron.J. $71,314$.

Garthwaite, K., Holdridge, D.B., and Mulholland, J.D.: 1970, Astron.J. 75,1133 .

Gutzwiller, M.C.: 1979, Astron. J. 84, 889.

Henrard, J.: 1973, Ciel et Terre 1.

Henrard, J.: 1979, Celest. Mech. 19,337. 
Henrard, J.: 1980, Celest. Mech. 22, 335.

Henrard, J.: 1981, Celest. Mech. 25, 417.

Kinoshita, H.: 1982a, Proceedings of the Conference on Analytical Methods and Ephemerides, Celest. Mech. 26, 71.

Kinoshita, H.: 1982b, these proceedings, 245-255.

Kubo, Y.: 1982, Proceedings of the Conference on Analytical Methods and Ephemerides, Celest. Mech. 26, 97.

Lestrade, J.F. et a1.: 1982, these proceedings, 217-225.

Schmidt, D.S.: 1980; Celest. Mech. 21, 163.

Standaert, D.: 1982a, Proceedings of the Conference on Analytical Methods and Ephemerides, Celest. Mech. 26, 113.

Standaert, D.: 1982b, these proceedings, 265-266.

Standaert, D.: 1982c, submitted for publication in Celest. Mech. 